\title{
Endoscopic pancreatic balloon sphincteroplasty for difficult to treat pancreatic stones and strictures: experience in 80 patients $\square$
}

\section{다(1) $(9)$}

\author{
Authors \\ Rinkesh Kumar Bansal, Gaurav Kumar Patil, Rajesh Puri, Narendra S. Choudhary, Saurabh R. Patle, Zubin D. Sharma, \\ Randhir Sud
}

Institution

Institute of Digestive and Hepatobiliary Sciences,

Medanta - The Medicity, Gurugram, Haryana, India

submitted 14.3.2017

accepted after revision 24.7.2017

Bibliography

DOI https://doi.org/10.1055/s-0043-119752 |

Endoscopy International Open 2017; 05: E1229-E1234

(c) Georg Thieme Verlag KG Stuttgart · New York

ISSN 2364-3722

Corresponding author

Rajesh Puri, MD DNB, Institute of Digestive and

Hepatobiliary Sciences, Medanta - The Medicity, Sector 38,

Gurugram, Delhi NCR, India, PIN 122001

Fax: +91-12-44834445

purirajesh70@gmail.com

\section{ABSTRACT}

Background and aim There is paucity of data about endoscopic pancreatic sphincteroplasty (EPS) after endoscopic pancreatic sphincterotomy (EPST) in the treatment of chronic pancreatitis. The aim of this study was to establish the indications for EPS, complications related to it, and to examine its effectiveness in managing chronic pancreatitis after a year of follow-up.

Methods We evaluated the safety and efficacy of pancreatic balloon dilation coupled with sphincterotomy for the treatment of chronic pancreatitis. The technical success rate of balloon dilation, stone clearance, frequency of pancreatic stenting, and procedure-related adverse events were recorded.

Results Out of 580 patients who underwent pancreatic endotherapy between July 2014 and February 2016, 80 patients underwent EPS. The mean age of these 80 patients was $34 \pm 11$ years, and $80 \%(n=64)$ were males. The common indications were removal of large radiolucent stones in 31 patients; unyielding radiopaque stones post extracorporeal shock wave lithotripsy (ESWL) in 20 patients, and pancreatic duct stricture combined with stones in 29 patients. EPS could be successfully completed in $98.75 \%$ of patients. Complete ductal clearance in a single session was achieved in only 25 patients, while 26 patients required two sessions. There were two adverse events of pain requiring admission for more than 24 hours and one procedure related bleeding, all of which were managed conservatively. The patients had an average follow-up of 8 months (6-12 months) and all the patients were pain free.

Conclusions Endoscopic pancreatic sphincteroplasty is a relatively safe procedure with a low incidence of complications and a high rate of treatment success.

\section{Introduction}

Chronic pancreatitis (CP) is a progressive fibro-inflammatory disease of the pancreas, characterized by irreversible pancreatic damage, and formation of pancreatic duct stricture and/or parenchymal stone, which leads to abdominal pain along with the presence of exocrine and endocrine insufficiency [1,2]. In the majority of patients with $\mathrm{CP}$, the presenting symptom is abdominal pain and often requires intervention either endoscopic or surgical $[3,4]$. The mechanisms of pain are multifactorial, but main pancreatic duct (MPD) obstruction due to stricture and or stone formation leading to ductal hypertension is one of the significant contributing factors [5-7].

Relief from abdominal pain is the main therapeutic goal and requires MPD clearance by endoscopic or surgical intervention. Endoscopic pancreatic sphincterotomy (EPST) followed by balloon or Dormia basket assisted stone extraction is usually successful when stones are of small size $(<5 \mathrm{~mm})$ and located in the head or proximal body region of the MPD [8]. However, the presence of multiple MPD stones, large radiolucent stones, and the presence of MPD strictures with impacted stones offer a significant challenge to the endoscopist. These can be mana- 
ged in two ways: either by decreasing the stone size or by enlarging the ampullary orifice [9].

Decreasing the stone size by breaking large stones into small pieces followed by easy extraction through the papilla can be done using mechanical lithotripsy, intraductal electrohydraulic lithotripsy (EHL) or extracorporeal shock wave lithotripsy (ESWL) [10]. ESWL is the most commonly used method which works on the principle of concentrating focused shock waves on stones, leading to stone fragmentation into small pieces, which can be retrieved endoscopically [15]. The success rate for pancreatic duct clearance varies from $37.5 \%$ to $100 \%$ and pain relief can be achieved in $79-100 \%$ of patients $[11,12]$. Tandan et al. reported $76.2 \%$ complete ductal clearance after three sessions of ESWL [13]. Radiopaque stones are easy to target under fluoroscopy but radiolucent stones need either ultrasound-guided shock wave lithotripsy or injection of contrast through the naso-pancreatic catheter [13].

An alternative method is endoscopic balloon sphincteroplasty (EPS) of the papilla, which consists of enlarging the papillary orifice followed by extraction of calculi of size $>5 \mathrm{~mm}$.

This technique can reduce the need for multiple ESWL sessions and ease the extraction of radiolucent stones which cannot be targeted by ESWL. However, there is limited literature with regard to this technique [14].

Today, EPST combined with ESWL serves as the cornerstone of endoscopic therapy. We incorporated the role of EPS accompanied by EPST in the treatment of CP and aimed to establish the indications for EPS, the complications related to it, and to examine its effectiveness in managing chronic pancreatitis.

\section{Methodology}

This retrospective study was conducted at a tertiary care institute in Gurgaon, India over a period of 19 months from July 2014 to February 2016. Data from 80 patients who underwent EPS was evaluated.

Patients with chronic pancreatitis and with abdominal pain as the presenting symptom were considered after they had failed medical management which consisted of complete avoidance of alcohol and smoking, use of adequate dose of analgesics, pancreatic enzyme supplementation and antioxidants. The frequency, duration, nature, intensity of pain, and need for analgesics were recorded. Frequency was assessed as the number of total pain episodes per week. The duration of each pain episode was recorded in minutes. The nature of pain, either continuous or intermittent, was noted and its intensity was recorded on a visual analogue scale. A scale of 0 to 10 was used where 0 indicated no pain, and 10 indicated severe, unbearable, continuous pain. A score of 0 to 1 was taken as no pain, 2 to 3 as mild pain, 4 to 6 as moderate pain, and 7 or more as severe pain. The number of analgesics consumed, both oral and parenteral, was noted. Endocrine manifestation was diagnosed if there was a prior history of diabetes mellitus and a requirement for oral hypoglycemic and/or insulin. In the absence of prior history, endocrine manifestation was diagnosed if fasting blood glucose was $>126 \mathrm{mg} / \mathrm{dL}$ and/or HbA1c levels were $>6.5 \%$. Exocrine manifestation was defined when there was a history of steatorrhea, defined as the presence of oil droplets in stool or the passage of large volume, foul smelling, pale, greasy stools.

EPS was considered in patients with large MPD stones, large radiolucent stones with a pancreatic head stricture, and those who failed EPST and conventional balloon retrieval or ESWL. A large MPD stone was defined when the stone size was $>5 \mathrm{~mm}$.

ESWL was performed in all of the patients before endotherapy. A third-generation electromagnetic lithotripter (Siemens) was used to deliver a maximum of 5000 shocks per session. An intensity of $15-16 \mathrm{kV}$ with a frequency of 90 shocks per minute was used for fragmentation. Repeat sessions were carried out after 2 weeks until the stone fragments were $<3 \mathrm{~mm}$ in diameter. ESWL failure was defined when there was less than $50 \%$ reduction in stone size after three sessions. The exclusion criteria were as follows: isolated tail calculi, extensive pancreatic duct calculi, multiple MPD strictures, associated pancreatic head mass or pseudocyst, coagulopathy (international normalized ratio $\geq 1.5$ ), significant thrombocytopenia (platelets $<50000 / \mu \mathrm{L}$ ), acute cholangitis or pancreatitis, malignant biliary strictures, or failure to give informed consent to the procedure.

Anticoagulant and antiplatelet agents were withheld for 5 days before the procedure. The procedure was done under sedation (intravenous administration of propofol) given by the anesthetist. The study was approved by the institutional review board (IRB).

EPST was performed using a side viewing endoscope (TJF180V; Olympus, Tokyo, Japan). Selective cannulation of the MPD was achieved using a sphincterotome (Ultratome XL; Boston Scientific, United States) and a 0.032/260 J-tip guidewire (Terumo wire). After obtaining a pancreatogram, a standard pancreatic EPST was performed using the sphincterotome. Biliary sphincterotomy was not done in these patients.

After EPST, MPD stone retrieval was attempted using a stone retrieval balloon or Dormia basket or both. If stone extraction failed, then EPS of the papillary orifice was performed. Using a 0.025 -inch guidewire (Vizyglide, Olympus) a 6,8 or $10 \mathrm{~mm}$ diameter Hurricane RX balloon (Boston Scientific Corp, United States) was inserted over the prepositioned guidewire and positioned across the papilla. The size of the balloon was decided according to the MPD size in the pancreatic head, i. e. proximal MPD. Subsequently, the balloon was inflated slowly (over 1 minute) with diluted contrast starting from $6 \mathrm{~mm}$ until the waist disappeared (the pressure was maintained not to exceed $8 \mathrm{~atm}$ ). Disappearance of the balloon waist along with achievement of the desired pressure was taken as complete dilation. After complete dilation, extraction of MPD stones was done using a stone retrieval balloon or Dormia basket. When complete ductal clearance was achieved, all of the patients underwent $10 \mathrm{~F}, 7 \mathrm{~cm}$ plastic stenting of the MPD. MPD stenting was done with the intention of preventing post procedure pancreatitis due to edema and the likelihood of smaller residual stones. All patients were observed for 24 hours after the procedure. They were followed up after 3 months with a repeat pancreatogram.

The primary study end point was the rate of complete ductal clearance. Complete stone removal was defined as the absence 
- Table 1 Baseline characteristics of patients with pancreatic sphincteroplasty.

Age, mean $\pm S D$ (range), years

$34 \pm 11(15-60)$

$\operatorname{Sex}(M / F), n$

64:16

Radiolucent stone/radiopaque stone/stricture with stone, $\mathrm{n}$

$31: 20: 29$

Abdominal pain/weight loss/endocrinal manifestations/exocrinal manifestations, n

$80: 40: 16: 10$

Duration of symptoms, median (range), months

$12(2-30)$

Cannulation of major papilla/minor papilla, $\mathrm{n}$

$52: 28$

Stone size, median (range), $\mathrm{mm}$

$8(5-15)$

Complications, n

3 (abdominal pain 2, bleeding 1 )

Follow-up, median (range), months

$8(6-12)$

of any filling defect during a final pancreatogram performed endoscopically or when more than $90 \%$ of the stone volume was cleared. Technical success was defined as the ability to retrieve all pancreatic duct stones.

Secondary study end points were the total number of endoscopic retrograde cholangiopancreatography (ERCP) sessions required and the occurrence of adverse events such as abdominal pain, post-ERCP pancreatitis (PEP), bleeding, cholangitis or perforation within 72 hours after EPS. Patients were followed up for an average of 8 months (6-12 months) post endotherapy.

Each adverse event was defined as per standard definitions. PEP was defined as continued abdominal pain 24 hours after ERCP accompanied by a greater than threefold increase in serum amylase levels above the upper limit of normal. Bleeding was defined as a drop in hemoglobin more than $2 \mathrm{~g} / \mathrm{dL}$ and cholangitis was defined as an increased temperature exceeding $38^{\circ} \mathrm{C}$ for more than 24 hours with cholestasis.

\section{Statistical analysis}

Data is presented as mean \pm SD or median (range). In subgroup analyses, the $x^{2}$ test or Fisher's exact test were used for categorical variables; Student's $t$ test was used for parametric data and the Mann-Whitney test was used for nonparametric data for comparison between two groups. Analyses were performed using SPSS 19.0 (SPSS Inc., Chicago, Illinois, United States). A two-tailed $P$ value less than 0.05 was considered to be statistically significant.

\section{Results}

An aggregate of 580 patients underwent pancreatic endotherapy between July 2014 and February 2016 at our center; out of these, 80 patients experienced EPS on an intention to treat basis. Standard EPST and/or ESWL was performed in the other 500 patients. Amongst the patients subjected to EPS, the mean age was $34 \pm 11$ years (range $15-60$ years), $80 \%(n=64)$ of patients were male, while $20 \%(n=16)$ were female.

In spite of optimal medical management, pain was the presenting symptom in all of the patients. Endocrinal manifestations were noted in $20 \%$ of patients, $12 \%$ had exocrine manifestations and $50 \%$ of patients complained of weight loss. The mean duration of symptoms was 12 months (range 2-30 months). In total, $45 \%$ of patients $(n=36)$ had a history of significant alcohol consumption ( $>20 \mathrm{~g}$ daily), while $25 \%$ of patients $(n=20)$ were smokers. Pancreas divisum was present in $12.5 \%(n=10)$, while $42.5 \%$ of patients $(n=34)$ had idiopathic calcific pancreatitis ( $\vee$ Table 1$)$.

Indications for EPS were removal of large radiolucent stones in 31 patients, unyielding radiopaque stones post ESWL in 20 patients, and pancreatic duct head stricture combined with stones in 29 patients.

Successful MPD cannulation was achieved with a sphincterotome in 52 of 80 patients and the remaining 28 patients underwent minor papilla cannulation. Minor papilla cannulation was done in patients with pancreas divisum and in those where the major papilla could not be cannulated. All stones were occluding the MPD mainly in the head region; most of the patients had multiple stones, and the mean stone size was $8 \mathrm{~mm}$ (range 5$15 \mathrm{~mm})$.

After EPST, once stone extraction was unsuccessful with basket and/or balloon, EPS of the papillary orifice was carried out. The mean size of dilating balloon used was $10 \mathrm{~mm}$ and the stones were extracted using a retrieval balloon or Dormia basket in all of the patients ( $\triangleright$ Fig. 1, $\triangleright$ Video 1 ). In patients with MPD stones, complete ductal clearance was achieved in 25 patients in one session while 26 patients required two sessions ( $\triangleright$ Fig. 2 and $\triangleright$ Fig.3). There was no dislodgment of stone fragments into the side branches during EPS.

All cases of strictures required graded dilation with a mean of 2.5 sessions (maximum four sessions) ( $\mathbf{F i g} .4$ ). The interval between two sessions was 3 months. In one patient, the stricture did not dilate adequately after three sessions and surgery was required. Technical success was achieved in $98.75 \%$ of patients $(n=79)$.

When we compared all three groups, there were no significant differences in the rate of achieving ductal clearance; however, the number of ERCP sessions was higher in the group with strictures compared to those without it. Minor adverse events were noted in three patients. Post procedure abdominal pain was the predominant complaint in around $50 \%$ of patients and this settled in 24 hours apart from two patients who required admission for more than 24 hours and were managed conserva- 

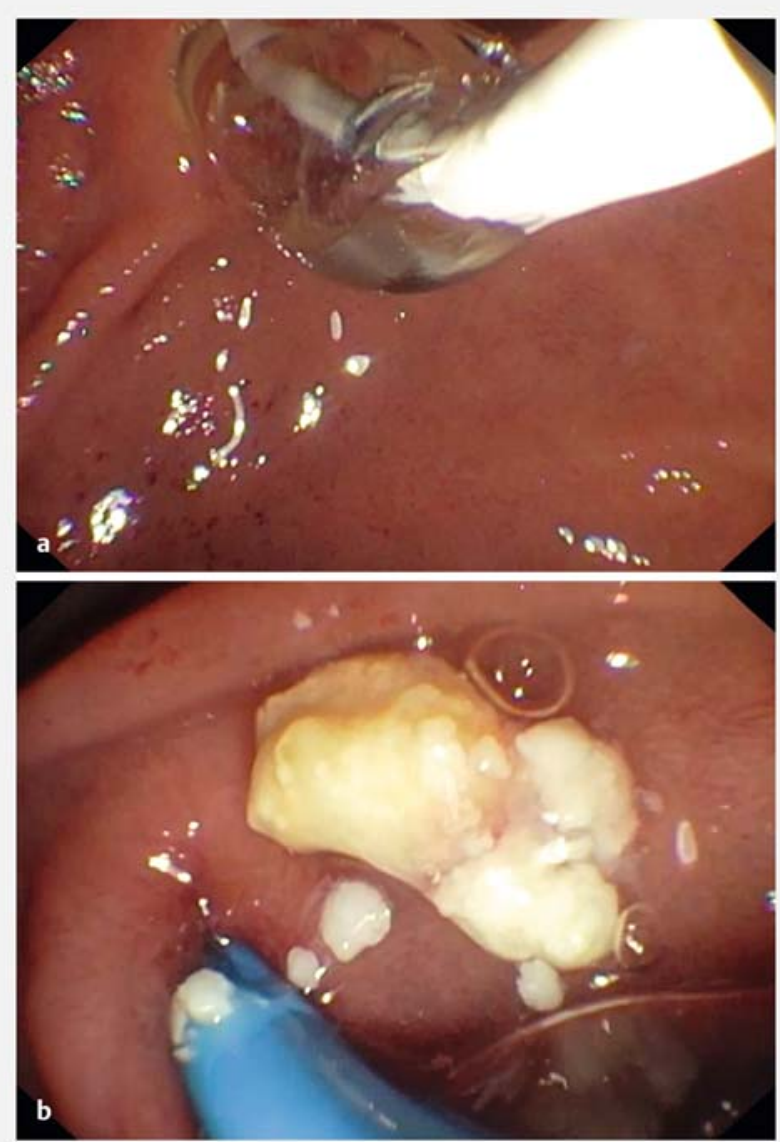

- Fig. 1 a Endoscopic image of balloon dilation (sphincteroplasty) of the papilla performed after sphincterotomy. $\mathbf{b}$ Endoscopic image of the extracted pancreatic calculi after sphincteroplasty.

tively. One patient developed minor bleeding during the procedure, which was managed by injection of epinephrine, and did not require blood transfusion or admission. No incidence of post-ERCP pancreatitis, cholangitis or perforation was noted.



Video 1 Pancreatic necrosectomy using saline and hydrogen peroxide solution.

All patients were discharged on the same day as the procedure except three patients with adverse events who required 48 hours stay in the hospital. Follow-up ERCP was done after 3 months in all of the patients after complete clearance and the stent was extracted after documenting no residual stones or stricture on pancreatogram.

The mean follow-up was 8 months (range 6-12 months) and all patients were asymptomatic until the last follow-up. Restenting was not required in any patient.

\section{Discussion}

Irrespective of the underlying etiology for $\mathrm{CP}$, intraductal pancreatic stones are pathognomonic and occur in the majority of patients. Stones are considered to be a consequence of the disease, and cause ongoing obstruction of outflow from the pancreas, leading to recurrent attacks of abdominal pain [15]. Today, EPST serves as the cornerstone of endoscopic therapy.
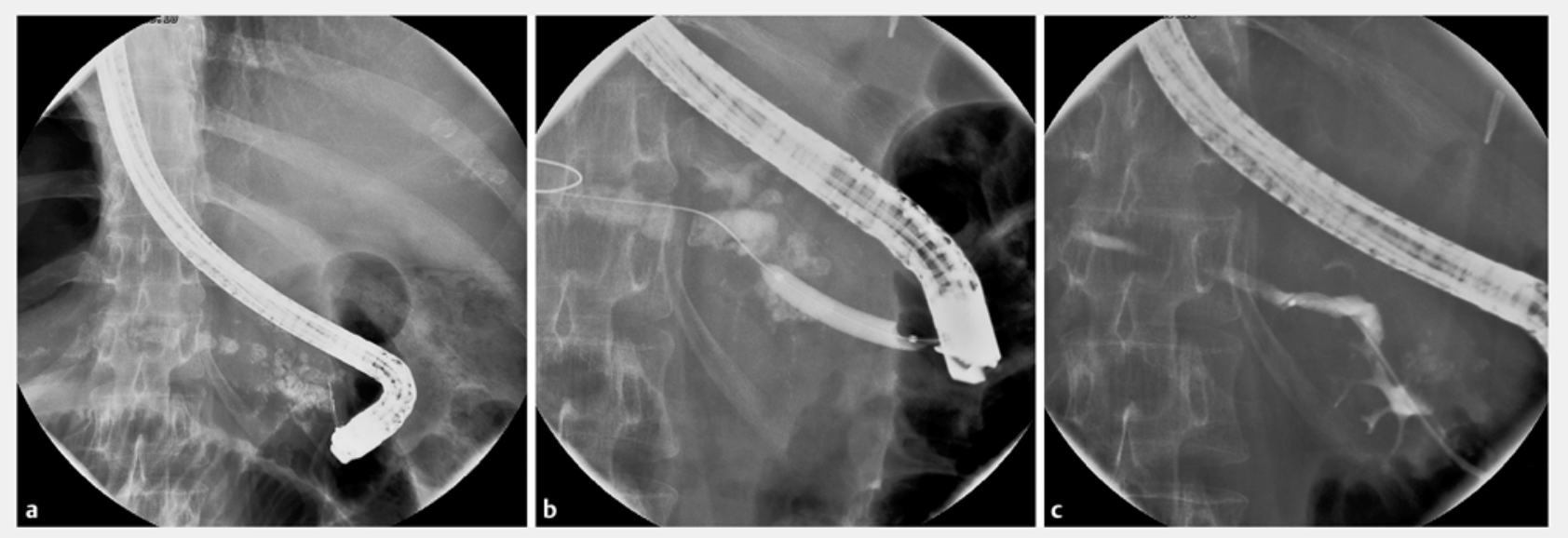

- Fig. 2 a Pancreatogram obtained endoscopically revealed a grossly dilated pancreatic duct along with multiple radiopaque calculi within. b Pancreatogram showing balloon dilation. c Follow-up pancreatogram showing mildly dilated duct without any stone. 

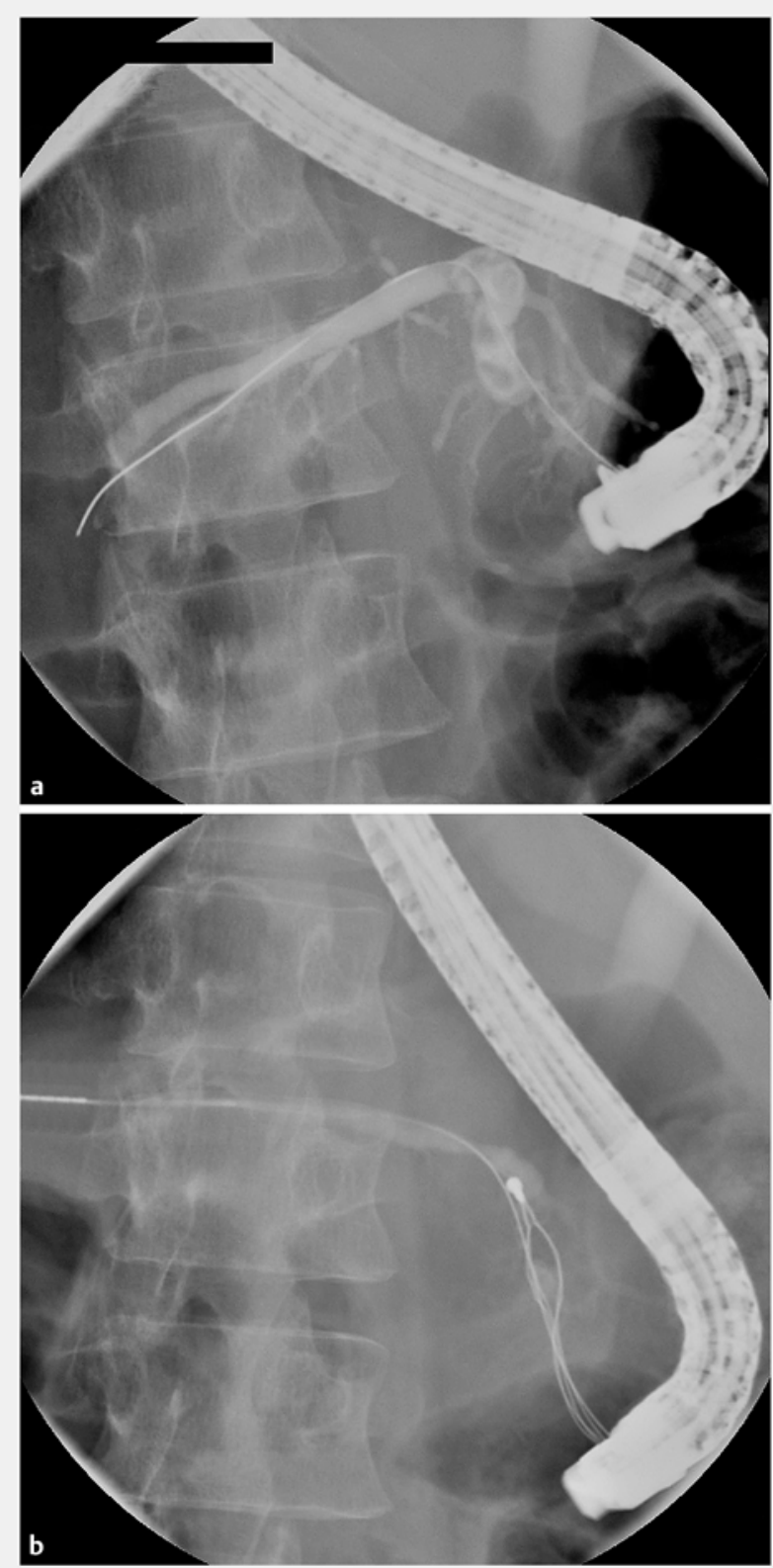

- Fig. 3 a Pancreatogram obtained endoscopically showing dilated pancreatic duct along with multiple large radiolucent calculi in head region. b Pancreatogram showing clearance of duct with Dormia basket after balloon dilation.

MPD stone removal is sometimes difficult endoscopically, even in post ESWL patients. EPST is often required after repeated sessions of ESWL. The majority of stones are radiopaque and can be targeted by ESWL followed by endoscopic removal [16]. On the other hand, radiolucent stones present a more challenging task, especially when they are large. The amount of calcium determines the hardness and therefore radiolucent stones tend to be softer than radiopaque stones [15]. We feel that EPST followed by EPS and balloon clearance would clear the MPD more effectively because of the accompanying strictures and ductal calculi.
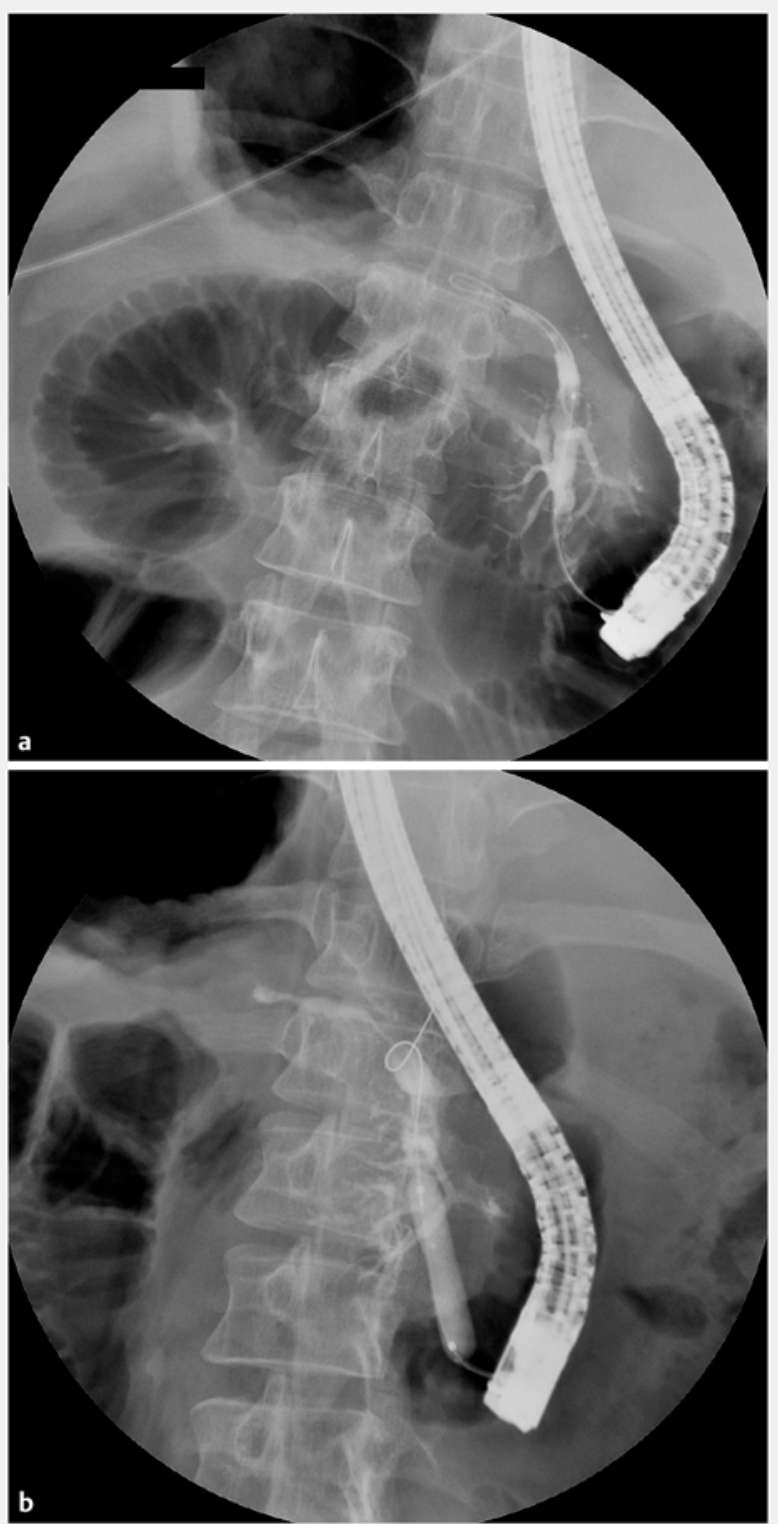

- Fig. 4 a Pancreatogram showing head stricture and dilated pancreatic duct distally. $\mathbf{b}$ Pancreatogram showing balloon dilation.

Small stones can be managed by EPST and ESWL, whereas large radiolucent stones present a therapeutic challenge. In our study, we performed successful EPS and clearance in all of the cases of large radiolucent stones and thereby obviated the need for placement of a naso-pancreatic catheter for ESWL or surgery. Large radiopaque stones were also managed effectively with EPS. In total, $50 \%$ of patients with a MPD stone required only one session of EPS, while the rest required two sessions. Complete clearance was achieved in all of the patients. In a study by Maydeo et al., balloon sphincteroplasty led to successful retrieval of large radiolucent stones in all four patients in a single session [14]. Suga et al. described balloon dilatation, without sphincterotomy, as a technique for retrieval of radiopaque MPD stones after fragmentation of the stone by ESWL [16]. 
In a large Indian cohort of 2779 patients with large MPD calculi, Tandan et al. demonstrated $80.1 \%$ complete ductal clearance and two-thirds of the patients required three sessions of ESWL. Our results with EPS appear promising in terms of number of ERCP sessions as well as the rate of achieving complete ductal clearance.

We also demonstrated successful clearance with EPS in patients with MPD head strictures with stones, although it required graded dilatation, hence a greater number of session was required than in those with stones alone. The average number of sessions required per patient was 2.5 , and a maximum of four sessions was needed to achieve complete clearance. One patient, who had insignificant dilatation after three sessions, was recommended for surgery. In our series, all of the patients had strictures in the head region, so EPS provided dual benefits; first, it accomplished papillary dilatation, second, it also produced simultaneous stricture dilatation.

EPS has the potential for adverse events such as abdominal pain, bleeding, retroduodenal perforation, cholangitis, or pancreatitis; however, we encountered adverse effects in only three of our patients (3.75\%), which were minor and were managed conservatively. Maydeo et al. reported adverse events in $50 \%$ of patients, including mild pancreatitis in one patient and minor bleeding in another; though they were managed conservatively. We performed sphincteroplasty by hurricane balloon while they used a controlled radial expansion balloon, which is more risky in comparison to a hurricane balloon. We advocate that EPS as a relatively safe procedure, though it requires further study in a large population.

The limitations of this study were its retrospective nature and short follow-up. Follow-up of an average 8 months (maximum 12 months) is not an adequate period to assess the response to any treatment in patients with CP. A longer followup period would be needed to assess the response to treatment in terms of pain relief as well as the effects on endocrine and exocrine functions.

In conclusion, endoscopic pancreatic sphincteroplasty is a relatively safe procedure with a low complication rate and a significant treatment success rate, thus, it can be used to successfully manage difficult to treat pancreatic duct stones and strictures in combination with endoscopic pancreatic sphincterotomy. We advocate that this modality should be used in a prospective manner and in a large population, so that the results can be validated.

Competing interests
References

[1] Etemad B, Whitcomb DC. Chronic pancreatitis: diagnosis, classification, and new genetic developments. Gastroenterology 2001; 120: $682-707$

[2] Bhasin DK, Singh G, Rana SS et al. Clinical profile of idiopathic chronic pancreatitis in North India. Clin Gastroenterol Hepatol 2009; 7: $594-$ 599

[3] Midha S, Khajuria R, Shastri S et al. Idiopathic chronic pancreatitis in India: phenotypic characterization and strong genetic susceptibility due to SPINK1 and CFTR gene mutations. Gut 2010; 59: 800-807

[4] Witt H, Apte MV, Keim V et al. Chronic pancreatitis: challenges and advances in pathogenesis, genetics, diagnosis, and therapy. Gastroenterology 2007; 132: 1557-1573

[5] Cremer M, Devière J, Delhaye M et al. Stenting in severe chronic pancreatitis: results of medium-term follow-up in seventy-six patients. Endoscopy 1991; 23: 171-176

[6] Delhaye M, Vandermeeren A, Baize M et al. Extracorporeal shock wave lithotripsy of pancreatic calculi. Gastroenterology 1992; 102: 610620

[7] Farnbacher M], Schoen C, Rabenstein T et al. Pancreatic duct stones in chronic pancreatitis: criteria for treatment intensity and success. Gastrointest Endosc 2002; 56: 501-506

[8] Maydeo A, Soehendra D, Reddy N et al. Endotherapy for chronic pancreatitis with intracanalar stones. Endoscopy 2007; 39: 653-658

[9] Bhasin DK, Rana SS. Extracorporeal shock wave lithotripsy (ESWL) for large pancreatic stones: are these shocks worthwhile? Indian J Gastroenterol 2010; 29: 133-136

[10] Freeman ML. Mechanical lithotripsy of pancreatic duct stones. Gastrointest Endosc 1996; 44: $333-336$

[11] Guda NM, Partington S, Freeman ML. Extracorporeal shock wave lithotripsy in the management of chronic calcific pancreatitis: A metaanalysis. JOP 2005; 6: 6-12

[12] Tadenuma H, Ishihara T, Yamaguchi T et al. Long term results of extracorporeal shockwave lithotripsy and endoscopic therapy of pancreatic stones. Clin Gastroenterol Hepatol 2005; 3: 1128-1135

[13] Tandan M, Reddy DN, Santosh D et al. Extra corporeal shockwave lithotripsy and endotherapy for pancreatic calculi - a large single center experience. Indian J Gastroenterol 2010; 29: 143-148

[14] Maydeo A, Bhandari S, Bapat M. Endoscopic balloon sphincteroplasty for extraction of large radiolucent pancreatic duct stones (with videos). Gastrointest Endosc 2009; 70: 798-802

[15] Suga T, Kawa S, Horiuchi A et al. Endoscopic pancreatic sphincter balloon dilation for effective retrieval of pancreatic duct stone. J Gastroenterol Hepatol 2000; 15: 220 - 224

[16] Tandan M, Reddy DN. ESWL of large pancreatic calculi: a decades experience. United Eur Gastroenterol J 2014; 2: (Suppl. 01): A7 